\title{
Real life turnaround time of blood cultures in the clinical microbiology laboratory: results of the first Italian survey, May 2015
}

\author{
Fabio Arena,1,2 Marta Argentieri,2,3 Paola Bernaschi,2,3 Giacomo Fortina,2 \\ Vesselina Kroumova, 2,4 Esther Manso, 2,5 Pier Giorgio Montanera, ${ }^{2,6}$ Pierluigi Nicoletti, $^{2}$ \\ Patrizia Pecile, 2,7 Mario Rassu, 2,8 Gian Maria Rossolini, 1,2,7,9,10 Teresa Spanu,2,11 \\ Pierangelo Clerici, ${ }^{12}$ Carla Fontana ${ }^{2,13}$ \\ ${ }^{1}$ Department of Medical Biotechnologies, University of Siena, Siena; ${ }^{2}$ Gruppo di Lavoro Infezioni \\ Paziente Critico, Associazione Microbiologi CLinici Italiani (AMCLI); ${ }^{3}$ Laboratory Department, \\ Bambino Gesù Children's Hospital, Rome; ${ }^{4}$ Infection Control Unit, University Hospital "della \\ Carità", Novara; ${ }^{5}$ Laboratory of Microbiology, University-Hospital Agency, Ospedali Riuniti, \\ Umberto I-Lancisi-Salesi, Ancona; ${ }^{6}$ Microbiology, Aosta Regional Hospital, Aosta; ${ }^{7}$ Clinical \\ Microbiology and Virology Unit, Florence Careggi University Hospital; ${ }^{8}$ Microbiology and Virology \\ Unit, San Bortolo Hospital, Vicenza; ${ }^{9}$ Department of Experimental and Clinical Medicine, \\ University of Florence; ${ }^{10}$ Don Carlo Gnocchi Foundation, Florence; ${ }^{11}$ Institute of Microbiology, \\ Catholic University of the Sacred Heart, Agostino Gemelli Hospital, Rome; ${ }^{12}$ Microbiology Unit, \\ Azienda Socio Sanitaria Territoriale Ovest Milano, Legnano; ${ }^{13}$ Clinical Microbiology Laboratories, \\ Tor Vergata University Hospital, Rome, Italy
}

\section{Summary}

Background and aims: Blood culture (BC) results are essential to guide antimicrobial chemotherapy for patients with sepsis. However, $\mathrm{BC}$ is a time-consuming exam, which can take several days. Reducing

Correspondence: Fabio Arena, Department of Medical Biotechnologies, University of Siena, Santa Maria alle Scotte University Hospital, Viale Bracci, Siena 53100, Italy.

E-mail: arena_fabio@hotmail.it

Acknowledgments: authors would thank Eleonora Riccobono, Cosima Marturano and Martina Stevan for assistance with data collection.

Key words: Turnaround time; Sepsis; Rapid molecular test; Laboratory workflow.

Contributions: FA, CF, GF and GMR, study conception and design; FA, CF, MA, $\mathrm{PB}, \mathrm{EM}, \mathrm{PP}$ and TS, collection and assembly of data; FA, data analysis and interpretation; FA, CF and GMR, manuscript writing.

Conflict of interest: the authors declare no potential conflict of interest.

Received for publication: 5 July 2016.

Revision received: 22 August 2016.

Accepted for publication: 25 August 2016.

CCopyright F. Arena et al., 2016

Licensee PAGEPress, Italy

Microbiologia Medica 2016; 31:6127

doi:10.4081/mm.2016.6127

This article is distributed under the terms of the Creative Commons Attribution Noncommercial License (by-nc 4.0) which permits any noncommercial use, distribution, and reproduction in any medium, provided the original author(s) and source are credited.
BCs turn around time (TAT) could impact on multiple outcome parameters and TAT monitoring is an important tool for measurement of microbiology laboratory performance. The aim of this study was to provide an overview of BC TATs among Italian microbiology laboratories.

Materials and methods: Five laboratories collected and recorded, for a month period, date and time of the $\mathrm{BC}$ processing events. Cumulative TATs were analysed using the GraphPad software.

Results: Participating laboratories reported data from 302 sepsis episodes. The median time from when the BC system produced a positive signal until Gram-stain results were reported was 7.6 hours. A rapid molecular identification and antimicrobial susceptibility testing (AST) was performed in $26.5 \%$ of BCs. Mean TAT for identification report was significantly lower when a molecular approach was adopted (12 vs. 28.7 hours, $\mathrm{P}<0.001$ ). Similarly, results of the molecular AST were obtained more than 24 hours in advance compared with phenotypic AST (mean 13.2 vs. 47.6, $\mathrm{P}<0.001$ ). TATs from BC positivity of laboratories opened 7 days/week were not significantly lower than those of laboratories opened 6 days/week.

Conclusions: $\mathrm{BC}$ is a time-consuming exam, however, molecular identification and AST methods can drastically reduce time to results. The lack of difference between TATs observed for laboratories working 7 days/week and 6 days/week, coupled with a high rate of BCs turning positive during the night enable to conclude that the most urgent measure to reduce TATs is the expansion of laboratory regular duty hours.

\section{Introduction}

Sepsis is a severe disease associated with high morbidity and mortality (7). Although conflicting data exist, early administration of appropriate empirical antimicrobial therapy, coupled with supportive treatment, have been shown to have an important impact on outcome $(6,9,10,16)$. 
Blood culture (BC) is the cornerstone for aetiological diagnosis of septicaemia and results are important to shift from empiric to definitive antimicrobial chemotherapy. However, $\mathrm{BC}$ is a time-consuming exam, which can take longer than 48 hours.

Although robust data showing a significant correlation between timeliness of reporting blood culture results and mortality are lacking, it seems likely that the two outcomes are associated. However, well structured studies demonstrated that the more rapid is the laboratory data reporting, the lower is the patients length of stay (both in Intensive Care Unit and hospital) and the antimicrobial consumption (4).

Newly introduced technologies such as PCR-based methods, fluorescence in situ hybridization and MALDI-TOF have expedited the causative pathogen identification $(1,5,8,19)$. Also on the antimicrobial susceptibility testing (AST) scenario there are several chances to reduce the TAT: i) direct inoculation of automatic susceptibility testing instruments or use of young culture on agar media $(12,13,17,20)$, and ii) use of liquid culture or time lapse microscopy based technologies $(1,11,14)$.

Molecular methods performed directly on positive blood cultures (broths) are able to provide preliminary information on antimicrobial resistance genes presence and pathogens identification in a turnaround time (TAT) of approximately three hours, hence resulting in a dramatic change of the Clinical Microbiology Laboratory contribution to the management of septic patients $(2,3,15,18)$.

TAT is a key indicator of clinical laboratory performance and is used by many clinicians to judge the quality of the laboratory service. For this reason we implemented a Clinical Microbiology Laboratory Network (CMLN), including laboratories adopting new approaches for expediting ID and AST results, aimed to provide an updated overview of real-life blood culture TATs among Italian microbiology laboratories.

\section{Materials and Methods}

A CMLN, consisting of five large Italian laboratories referring to members of the Gruppo di Lavoro Infezioni Paziente Critico working group (belonging to Associazione Microbiologi CLinici Italiani) was created in April 2015. Three out of five laboratories were located within Hospitals with $\geq 1000$ beds, while two in Hospitals with $400-700$ beds.

CMLN Laboratories were asked to collect and record in a dedicated database, for a month period (1 to 30 May 2015), the date and time of the following $\mathrm{BC}$ processing events: i) laboratory check-in (the time when the $\mathrm{BC}$ was taken in charge by the laboratory staff); ii) $\mathrm{BC}$ positivity signal (instrument alarm); iii) Gram-stain microscopic examination completed and results successfully reported to clinical staff; iv) bacterial/fungal identification, obtained with rapid molecular methods other than MALDI-TOF or biochemical ID report; v) MALDI-TOF or biochemical bacterial/fungal ID report (including results obtained with lysis-filtration protocols, young culture processing and conventional overnight approach); vi) molecular detection of antimicrobial resistance markers, directly from BC liquid, report; vii) phenotypic AST final report (including results obtained with automated AST instruments or broth microdilution method using young or overnight culture).

Analysis was restricted to data regarding the first positive blood culture bottle from a set, for all consecutive, non-replicated, sepsis episodes occurred within the study period. BCs positive for contaminants (positivity of one bottle/set only for skin colonizing bacteria) were excluded from the analysis.

TATs were expressed in hours and the minimum, maximum, median, $25^{\text {th }}$ percentile and $75^{\text {th }}$ percentile values were calculated using the GraphPad software (GraphPad Software, Inc.) TATs were compared using the two-tailed t test.

The participating laboratories were also asked to provide full information on systems used for: BCs monitoring; conventional ID; molecu- lar pathogen ID from BC liquid and detection of resistance determinants markers; phenotypic AST; software used for results reporting and storage (laboratory information system). Moreover, the laboratories were asked to provide the number of $\mathrm{BC}$ exams performed in the previous year (2014) as indicator of their workload, together with details on opening days/week and hours/day.

\section{Results}

Among the participating laboratories, two out of five, processed positive BCs 7 days/week while the other three processed positive BCs 6 days/week. All participants used continuously monitoring blood culture systems.

During opening days, participating laboratories processed BCs over a timespan ranging from 9 to 15 hours with an intermittent processing schedule during opening hours. The majority of IDs were obtained by MALDI-TOF technology and phenotypic AST was performed mainly with automated AST systems (Table 1 and data not shown).

Overall, during the surveillance period, CMLN laboratories reported data from 302 sepsis episodes (range 41-91 episodes per Laboratory). The molecular ID and AST were performed for $80 \mathrm{BCs}(26.5 \%)$ and the phenotypic AST was performed in 277 cases (92\%). The mean and median times to positivity from check-in (Check-Pos) were 15.1 and 20.4 hours, respectively.

Among all laboratories, the median time from when the BC system produced a positive signal until Gram-stain results were reported (PosGram) was 7.6 hours, ranging from 0.2 to 86.4 hours (mean 10.3 hours). TAT analysis is summarised in Table 2 and Figure 1. Mean TAT for ID report was significantly lower when a molecular approach was adopted (12 vs. 28.7 hours, $\mathrm{P}<0.001$ ). Similarly, results of the molecular AST were obtained more than 24 hours in advantage comparing with phenotypic AST (mean 13.2 vs. 47.6, $\mathrm{P}<0.001$ ).

Cumulatively, TATs from BC positivity of Laboratories opened 7 days/week were not significantly lower than those of Laboratories opened 6 days/week with mean Pos-Gram: 11.5 vs. 9.1, mean Pos-ID: 30 vs. 27.9 and mean Pos-AST: 48 vs. 47.2 hours.

Regarding time to positivity, $49.3 \%$ of blood cultures turned positive during the night (from 20:00 to 8:00) and therefore were processed the following morning.

Interestingly, the TATs distribution for identification and molecular AST were very similar to that of Gram-stain report (Figure 1).

\section{Discussion and Conclusions}

Overall, the analysis of data from CMLN showed that the determination of phenotypic conventional AST is still a time-consuming process that requires a mean of 47.6 hours from $\mathrm{BC}$ positivity. However, the adoption of AST protocols based on the use of young culture or rapid broth enrichment protocols can lead to the obtainment of AST results within 12 hours.

The relevant advantage of use of MALDI-TOF ID is confirmed by the fact that mean TAT of ID anticipates by more than 20 hours the phenotypic AST result (mean TAT 28.7 vs. 47.6 hours).

Our analysis confirms that molecular ID and AST methods able to give rapid results from $\mathrm{BC}$ broth are breakthrough technologies that can dramatically reduce time ID and AST results (TAT 12 and 13.2 hours from BC positivity, respectively). However, due to the high cost of these technologies in comparison to conventional ID and AST, they can be adopted in a subset of selected cases only (in our surveillance, molecular ID and AST were performed in approximately a quarter of 
Table 1. Results obtained form laboratory tests.

\begin{tabular}{|c|c|c|c|c|c|c|c|}
\hline Centre & Beds & $\begin{array}{c}\text { BCs } \\
\text { in } 2014\end{array}$ & $\begin{array}{l}\text { N. positive } \\
\text { BCs, May } \\
2015\end{array}$ & $\begin{array}{l}\text { N. sepsis } \\
\text { episodes, } \\
\text { May } 2015\end{array}$ & $\begin{array}{l}\text { N. } \\
\text { molecular } \\
\text { AST }\end{array}$ & $\begin{array}{c}\text { System used } \\
\text { for molecular AST }\end{array}$ & $\begin{array}{l}\text { Laboratory } \\
\text { opening hours }\end{array}$ \\
\hline 1 & 607 & 14,356 & 97 & 42 & 8 & $\begin{array}{c}\text { GeneXpert } \\
\text { (Cepheid Sunnyvale, CA, USA) }\end{array}$ & $\begin{array}{l}\text { 8:00 to 20:00, } \\
7 \text { days/week }\end{array}$ \\
\hline 2 & 1644 & 22,000 & 400 & 91 & (A & $\begin{array}{c}\text { Eazyplex } \\
\text { Amplex BioSystems Giessen, Germany), } \\
\text { GeneXpert }\end{array}$ & $\begin{array}{l}\text { 7:00 to 19:00, } \\
6 \text { days/week }\end{array}$ \\
\hline 3 & 460 & 25,047 & 248 & 41 & 19 & $\begin{array}{c}\text { Film Array } \\
\text { (bioMérieux Marcy l'Etoile, France), } \\
\text { home brew PCR }\end{array}$ & $\begin{array}{l}\text { 7:30 to } 20: 00 \\
7 \text { days/week }\end{array}$ \\
\hline 4 & 1000 & 10,151 & 139 & 62 & 4 & GeneXpert & $\begin{array}{l}\text { 7:00 to } 16: 00, \\
6 \text { days/week }\end{array}$ \\
\hline 5 & 1297 & 19,482 & 277 & 66 & 4 & $\begin{array}{c}\text { Verigene } \\
\text { (Nanosphere, Northbrook, IL, USA) }\end{array}$ & $\begin{array}{l}\text { 7:00 to } 22: 00, \\
6 \text { days/week }\end{array}$ \\
\hline
\end{tabular}

BC, blood cultures; AST, antimicrobial susceptibility testing.

Table 2. Results of turn around time analysis.

\begin{tabular}{lcccccc} 
& Check-Pos & Pos-Gram & Pos-ID & Pos/IDM & Pos-ATBM P0s-ATB \\
Number of values & 302 & 302 & 222 & 80 & 80 & 277 \\
Range & $1.7-109.4$ & $0.2-86.4$ & $1-132$ & $1-38.4$ & $1-41.5$ & $9.6-138.4$ \\
\hline Median & 15.1 & 7.6 & 23.5 & 7.9 & 8.6 & 41.2 \\
Mean & 20.4 & 10.3 & 28.7 & 12 & 13.2 & 47.6 \\
\hline
\end{tabular}

Check-Pos, Check-in to positivity; Pos-Gram, positivity to Gram-stain; Pos-IDM, positivity to molecular pathogen identification; Pos-ID, positivity to pathogen identification; Pos-ASTM, positivity to molecular detection of resistance markers; Pos-AST, positivity to phenotypic antimicrobial susceptibility testing.

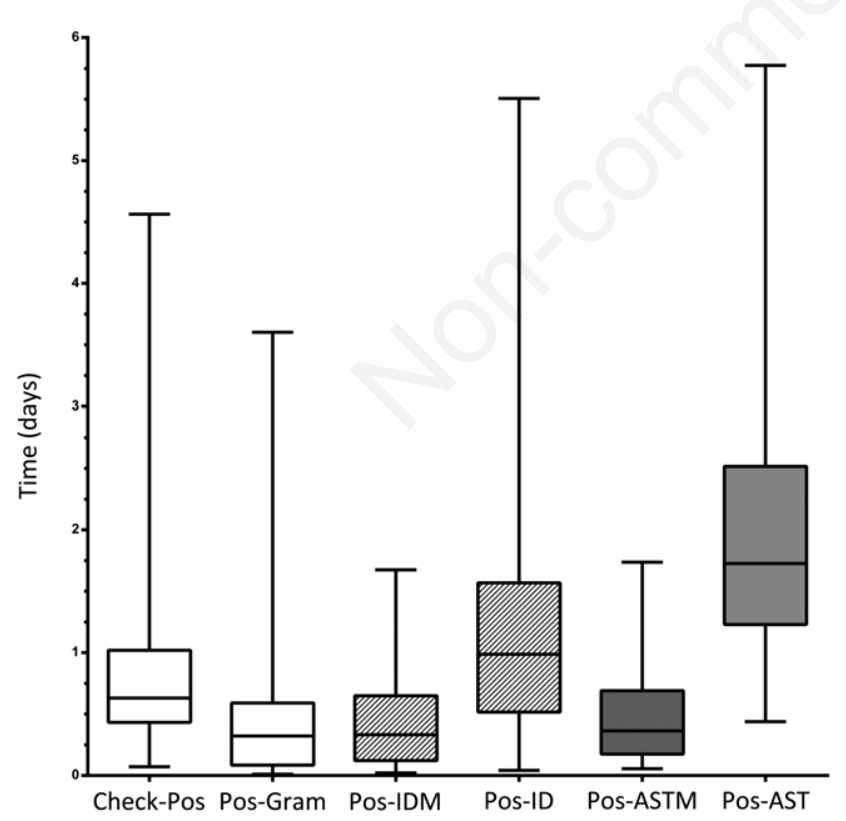

Figure 1. Time intervals used for the turnaround time analysis (in days): from check-in to positivity (Check-Pos); from positivity to Gram-stain reporting (Pos-Gram); from positivity to molecular pathogen identification reporting (Pos-IDM); from positivity to pathogen identification reporting (Pos-ID); from positivity to molecular detection of resistance markers reporting (Pos-ASTM); from positivity to phenotypic antimicrobial susceptibility testing results (Pos-AST). Boxes indicate the range from 25 to $75 \%$. sepsis episodes). Overall, participating laboratories declared to process with molecular ID and AST the positive BCs obtained from patients admitted to Intensive Care Units, Neonatal Intensive Care Units and Haematology wars.

In all participating laboratories, even for fast molecular methods and Gram-stain, we found a significant delay of observed versus theoretical TATs. This phenomenon is probably related to the high proportion (49.6\%) of $\mathrm{BC}$ that turned positive outside of the regular duty hours, causing several hours delays in the analysis of positive BCs.

The lack of difference between TAT observed for laboratories working 7 days/week and 6 days/week enabled us to conclude that the most urgent measure to reduce TATs in microbiology is the progressive expansion of regular duty hours up to 24 hours/day.

One possible limitation of our study is that information on the preanalytical phase were not available for all laboratories, since storage and transport times are generally out of the laboratory's control. Therefore this phase was excluded from the analysis. However, even if the pre-analytical phase could influence the time elapsed from check-in to $\mathrm{BC}$ positivity, the time elapsed from $\mathrm{BC}$ positivity to the results of the various analytical steps is not expected to be biased by the conditions of the preanalytical phase and, in our opinion, these data can be reliably used as indicators of the performance of the laboratory workflow.

\section{References}

1. Arena F, Viaggi B, Galli L, Rossolini GM. Antibiotic susceptibility testing: present and future. Pediatr Infect Dis J 2015;34:1128-30.

2. Banerjee R, Teng CB, Cunningham SA, et al. Randomized trial of 
rapid multiplex polymerase chain reaction-based blood culture identification and susceptibility testing. Clin Infect Dis 2015;61: 1071-80.

3. Bork JT, Leekha S, Heil EL, et al. Rapid testing using the verigene gram-negative blood culture nucleic acid test in combination with antimicrobial stewardship intervention against gram-negative bacteremia. Antimicrob Agents Chemother 2015;59:1588-95.

4. Buehler SS, Madison B, Snyder SR, et al. Effectiveness of practices to increase timeliness of providing targeted therapy for inpatients with bloodstream infections: a laboratory medicine best practices systematic review and meta-analysis. Clin Microbiol Rev 2015;29: 59-103.

5. Chun K, Syndergaard C, Damas C, et al. Sepsis pathogen identification. J Lab Auto 2015;20:539-61.

6. de Groot B, Ansems A, Gerling DH, et al. The association between time to antibiotics and relevant clinical outcomes in emergency department patients with various stages of sepsis: a prospective multi-center study. Crit Care BioMed Central 2015;19:194.

7. Dellinger RP, Levy MM, Rhodes A, et al. Surviving sepsis campaign: international guidelines for management of severe sepsis and septic shock: 2012. Crit Care Med 2013;41:580-637.

8. Dubourg G, Raoult D. Emerging methodologies for pathogen identification in positive blood culture testing. Expert Rev Mol Diagn 2015;16:97-111.

9. Ferrer R, Artigas A, Levy MM, et al. Improvement in process of care and outcome after a multicenter severe sepsis educational program in Spain. JAMA 2008;299:2294-303.

10. Fitzpatrick JM, Biswas JS, Edgeworth JD, et al. Gram-negative bacteraemia; a multi-centre prospective evaluation of empiric antibiotic therapy and outcome in English acute hospitals. Clin Microbiol Infect 2016;22:244-51.

11. Fontana C, Favaro M, Minelli S, et al. A novel culturing system for fluid samples. Med Sci Monit 2009;15:BR55-60.

12. Idelevich EA, Schüle I, Grünastel B, et al. Acceleration of antimicro- bial susceptibility testing of positive blood cultures by inoculation of Vitek 2 cards with briefly incubated solid medium cultures. J Clin Microbiol. 2014;52:4058-62.

13. Kerremans JJ, Verboom P, Stijnen T, et al. Rapid identification and antimicrobial susceptibility testing reduce antibiotic use and accelerate pathogen-directed antibiotic use. J Antimicrob Chem 2008;61:428-35.

14. Kroumova V, Gobbato E, Macaluso P, et al. Preliminary indications for antibiotic susceptibility tests in less than six hour in positive blood cultures. Microbiol Med 2010;25:24-6.

15. Ledeboer NA, Lopansri BK, Dhiman N, et al. Identification of gramnegative bacteria and genetic resistance determinants from positive blood culture broths by use of the verigene gram-negative blood culture multiplex microarray-based molecular assay. J Clin Microbiol 2015;53:2460-72.

16. Levy MM, Dellinger RP, Townsend SR, et al. The surviving sepsis campaign: results of an international guideline-based performance improvement program targeting severe sepsis. Crit Care Med 2010;38:367-74.

17. Romero-Gómez M-P, Gómez-Gil R, Paño-Pardo JR, Mingorance J. Identification and susceptibility testing of microorganism by direct inoculation from positive blood culture bottles by combining MALDI-TOF and Vitek-2 Compact is rapid and effective. Elsevier Ltd; 2012. pp 1-8.

18. Salimnia H, Fairfax MR, Lephart PR, et al. Evaluation of the FilmArray blood culture identification panel: results of a multicenter controlled trial. J Clin Microbiol 2016;54:687-98.

19. Schneiderhan W, Grundt A, Worner S, et al. Work flow analysis of around-the-clock processing of blood culture samples and integrated MALDI-TOF mass spectrometry analysis for the diagnosis of bloodstream infections. Clin Chem 2013;59:1649-56.

20. Verroken A, Defourny L, Lechgar L, et al. Reducing time to identification of positive blood cultures with MALDI-TOF MS analysis after a 5-h subculture. Eur J Clin Microbiol Infect Dis 2014;34:405-13. 